\title{
Continuous Functional Activity Monitoring Based on Wearable Tri-axial Accelerometer and Gyroscope
}

\author{
Yuting Zhang \\ Department of Electrical and Computer Engineering \\ Boston University \\ Boston, USA \\ ytzhang@bu.edu
}

Stacey Markovic

Department of Biomedical Engineering

Boston University

Boston, USA

markovic@bu.edu

\author{
Inbal Sapir \\ Department of Physical Therapy and Athletic Training \\ Boston University \\ Boston, USA \\ ssinbal@bu.edu
}

\author{
Robert C. Wagenaar \\ Department of Physical Therapy and Athletic Training \\ Boston University \\ Boston, USA \\ wagenaar@bu.edu
}

\author{
Thomas D.C. Little \\ Department of Electrical and Computer Engineering \\ Boston University \\ Boston, USA \\ tdcl@bu.edu
}

\begin{abstract}
Given the growing number of elderly people and patients diagnosed with Parkinson's disease, monitoring functional activities using wearable wireless sensors can be used to promote the Quality of Life and healthier life styles. We propose a novel and practical solution using three small wearable wireless Functional Activity Monitor (FAM) sensors and a smartphone to store, transmit, analyze and update data. Three sensors, each composed of a tri-axial accelerometer and a triaxial gyroscope, are attached to the chest and both thighs. A computationally efficient signal processing algorithm is designed to accurately measure tilting angles. A continuous activity recognition algorithm is developed using a decision tree based on time series data and spectrum analysis; this algorithm can identify activities of daily life in three general categories: (1) postures such as standing, sitting, and lying; (2) locomotion such as walking; and (3) transitions such as sit-to-stand and stand-tosit. The results show an accurate angle measurement compared to the motion capture system Optotrak 3020 and a reliable detection of all activities with sensitivity at least $96.2 \%$ compared to video recordings.
\end{abstract}

Keywords-accelerometer; gyroscope; mobility monitoring; signal processing; drift compensation; activity recognition

\section{INTRODUCTION}

The aging of the population and the increase of chronic diseases and Parkinson's disease have already had a major impact on most western health care systems [1], and will have continuously increasing effects in the future. In the United States, 12.4 percent of the population were adults 65 and older in 2005 [2], and it is estimated that about 1 in 5 will be elderly

This work is supported by the Wallace H. Coulter Translational Research Partnership Awards. by the year 2030. The need for personal assistance with daily living activities increases with the age, therefore, the quantification of daily physical activities is essential in the evaluation of the functional ability and the Quality of Life of subjects with limited mobility and in rehabilitation. Aminian et al. show that a slower gait, a reduction of daily walking distance and a decrease in the number of walking episodes are indicators of disease progression [3]. Elderly people and patients with chronic pain, Parkinson's disease and stroke also have a high risk of falling.

There are several methodologies used in monitoring functional activities such as questionnaires [4], video recordings [5], and sonic, magnetic and optical motion capture systems [6]. However, these systems have various limitations in accurately mobile monitoring daily activities in the home and community setting. The technology of Micro Electro Mechanical Systems (MEMS) boosts the development of miniature and low powered inertial sensors, accelerometers and gyroscopes, to analyze human movement based on kinematics. The prevalence of wireless communication techniques and smartphones enable physicians and patients to continuously monitor functional activities through wireless wearable sensors.

Devices using accelerometers alone to measure angles are inaccurate when angles are large or in accelerating movements [7]. The gyroscope is largely adopted to improve the accuracy of angle measurements [8]. The intrinsic drift produces large error after numerical integrating angular rates to angles. The uses of Kalman filters, wavelet transforms and neural networks have significantly reduced the drift [7][9][10], but these 
algorithms are complex to be implemented in real-time online due to the computational power and energy consideration of the smartphone.

The objective of this study is to develop a FAM system that can (1) continuously measure body tilting angles in an accurate and computationally efficient manner, (2) record and analyze functional activities, and (3) further provide accurate real-time feedback via a smartphone in the home and community setting. It is hypothesized that by means of three FAM sensors attached to the chest and both thighs, the signal processing algorithm can accurately measure the movement's amplitude of the body segments; and the activity recognition algorithm can accurately detect postures (sitting, standing, lying), locomotion (walking) and transitions in a window of one second, and provide a summary of durations of daily activities to quantify the level of activity. The signal processing and recognition algorithms are computationally efficient to be executed real-time online on the smartphone instead of traditionally post hoc on a personal computer. Furthermore, this system can offer options to detect step/stride frequency and to quantify level of activity to give different perspectives in diagnosis, prognosis, and responsiveness of treatments. This paper is organized as follows: methods including measuring system, study design and algorithms are explained in section II; results are presented in section III; and a discussion is in section IV.

\section{METHODS}

\section{A. Measuring System}

The FAM sensor is the Inertial Measurement Unit 6 Degree of Freedom version 4 (IMU-6DOF-v4) fabricated by SparkFun Electronics, Boulder, CO. It is a two-printed circuit board (PCB) unit at the size of $50 \mathrm{~mm} \times 40 \mathrm{~mm} \times 15 \mathrm{~mm}$, small and affixable to the human body. The kinematic sensors are composed of one triple-axis accelerometer (Freescale, MMA7260Q, settable to $1.5 \mathrm{~g}, 2 \mathrm{~g}, 4 \mathrm{~g}$ or $6 \mathrm{~g}$ sensitivity) and two gyroscopes (InvenSense, IDG500, 500 degree/second) aligned to work as triple-axis. Three FAM sensors attached to the chest and both thighs measure angles and angular velocities in the vertical, frontal and saggital axes. The FAM sensor transmits data to the smartphone (AT\&T 8525) over a Bluetooth link. The smartphone stores data, analyzes data online, provides feedback to the user, and can further update results to the health care center through either an Internet or cellular connection.

For the purposes of measuring angles and monitoring functional activities accurately while reducing energy consumption to prolong the battery life, the sensitivity of accelerometer is set to its highest at $1.5 \mathrm{~g}$, the gyroscope is working at its full range, and the sampling frequency is set to $50 \mathrm{~Hz}$ in the configuration on the IMU development platform.

The Optotrak 3020 (Northern Digital Inc., Waterloo, ON, Canada) is employed as reference to test the accuracy of the angle measurements of FAM system. The Optotrak 3020, consisting of wired infrared light-emitting diodes (IREDs) captured by banks of cameras, is a widely used threedimensional motion capture system in laboratory settings in the field of movement analysis [11]. A video recording system consisting of two high definition cameras (HS 100P/PC,
Panasonic Inc. and VIXIA HG21, Cannon Inc.) is used as reference to test the activity recognition algorithm.

\section{B. Study Design}

Three studies demonstrated in Fig. 1 were conducted to develop and test the FAM system. The specific aim of the first study was to develop an accurate signal processing algorithm to measure static angles. The second study focused on testing its accuracy on measuring dynamic angles of oscillation. The third study enrolled 10 healthy young subjects to train and test the activity recognition algorithm. All studies were conducted in the Clinical Movement Sciences Lab, and written informed consents were obtained from all the subjects.

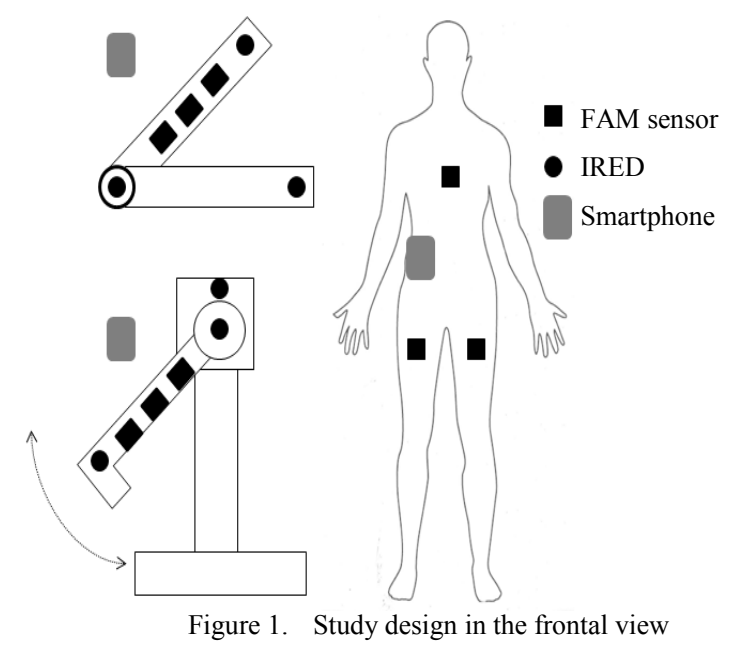

1) First study: The Digital Angle Protractor (Denali) was set to five fixed angles, $0^{\circ}, 30^{\circ}, 60^{\circ}, 90^{\circ}$, and $120^{\circ}\left(-60^{\circ}\right.$ in the FAM and Optotrak system), holding for 1 minute each. Three FAM sensors were attached to the tilting arm, and sensing data was collected by the smartphone and then analyzed. Three IREDs were placed on both arms and at the axis, and a bank consisting of three cameras was placed at a distance of three meters perpendicular to the angle protractor plane.

2) Second study: The Biodex system (SEMI, Toronto, ON), set to the passive mode, was programmed to produce consistent and precise oscillations at desired angles and frequencies [12]. Twenty oscillating trials were set at the amplitude of $120^{\circ}, 90^{\circ}$, $60^{\circ}, 30^{\circ}$ and $5^{\circ}$ at four different frequencies 80, 60, 40, 20 bits/min, and each trial lasted for 1 minute. Three FAM sensors were attached on the rotating arm. The first IRED was placed at the end of the moving arm, the second on the axis of rotation, and the third aligned vertically with the second in order to provide a reference segment. A bank of three cameras was placed perpendicular to the oscillation plane at a distance of five meters.

3) Third study: 10 healthy young subjects ( 5 females, 5 males, $23 \pm 3$ years old) performed functional activity trials, including (1) 1 trial of 10 transfers from standing to sitting and vice versa, (2) 1 trial of transfers from standing to sitting to lying down to sitting then back to standing, and (3) 15 trials of standing to walking to standing, 5 at each of the subject's preferred, slow, and fast speed. As the duration of transitions is naturally 2 or 3 seconds, to test our algorithms all postures and 
locomotion lasted for more than 10 seconds. Three FAM sensors were attached to the chest, right and left thigh respectively, and the video recording system was placed facing the subject.

\section{Signal Processing}

The key feature to detect forward leans to prevent falls is the chest angle versus the vertical axis. A computationally efficient signal processing algorithm illustrated in Fig. 2 is used in the FAM system to accurately measure body angles. This algorithm does not need to perform computationally expensive calculations needed for traditional methods such as complex presumptions and matrix multiplications in the Kalman filter or at least five layers of decomposition of time series into approximate and detail parts in the wavelet transforms.

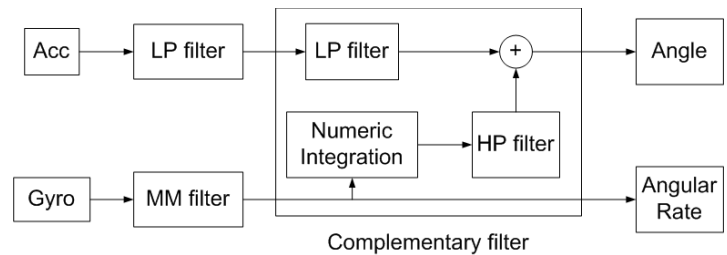

Figure 2. Signal processing flow

The accelerometer signals are filtered using a $2^{\text {nd }}$-order forward-backward digital low-pass Butterworth filter with a cutoff frequency at $3 \mathrm{~Hz}$ [13]. The angle from the accelerometer AccAngle is calculated from three orthogonal components $g_{x}, g_{y}$, and $g_{z}$ countering the gravity by an arctangent function as in (1), because the resolution of either arcsine or arccosine function varies significantly through the tilt range.

$$
\text { AccAngle }=\arctan \left(\frac{g_{z}}{\sqrt{g_{x}^{2}+g_{y}^{2}}}\right)
$$

Considering the frequency range of human movements, any filter with clear cutoff frequencies would severely attenuate important movement signals in its stopband, and thus cannot be applied to MEMS gyroscope signals. Instead, a median-mean filter as in (2) is designed to eliminate burst noise and outlier signals [14], where $x_{n}$ is the angular rate sensed by the gyroscope and $y_{n}$ is the filtered angular rate. The window width $2 \mathrm{~m}+1$ is selected carefully to preserve useful information and $\mathrm{m}$ is often set to be within the range of 3 to 5 .

$$
\begin{array}{r}
y_{m+1}=\operatorname{median}\left(\operatorname{mean}\left(x_{1}, x_{2}, \ldots x_{m}\right), x_{m+1},\right. \\
\left.\operatorname{mean}\left(x_{m+2}, x_{m+3}, \ldots x_{2 m+1}\right)\right)
\end{array}
$$

A complementary filter as in (3) is designed to compensate the gyroscope's drift by the accelerometer, working as a Proportional-Derivative controller where the angle measured by the accelerometer is the proportional part and the angular rate from the gyroscope is the $1^{\text {st }}$ order derivative part. The complimentary filter functions as the combination of a highpass filter and low-pass filter structured inside in Fig. 2. In (3) Angle is the angle output of the complimentary filter, GyroRate is the angular rate after median-mean filter and AccAngle is the angle calculated from the accelerometer, where $(n+1)$ is the corresponding data following $(n)$ in time series. The coefficients $H P F$ and $L P F$ usually sum to 1 and can be estimated by the Genetic Algorithm. In this study coefficients are trained and tested by angles measured by the Optotrak system.

$$
\begin{aligned}
\operatorname{Anlge}(n+1)= & H P F \\
& \times\left(\operatorname{Angle}(n)+\operatorname{GyroRate}(n+1) \times \frac{1}{F_{s}}\right) \\
& +\operatorname{LPF} \times \operatorname{AccAngle}(n+1)
\end{aligned}
$$

\section{Activity Recognition}

To identify postures, locomotion and transitions, a decision tree is built as in Fig. 3. Tilting angles of chest and both thighs are inputted to the decision tree and segmented by a sliding window of 1 second. The standard deviation (SD) of these three angles is calculated in every second. From training data, SD of $2^{\circ}$ is obtained as the threshold to decide static activities (postures) or dynamic activities (locomotion and transitions). The three mean angles in the same second are evaluated to further classify static postures into standing, sitting, and lying down; if none is identified, the posture will be labeled as an unidentified posture. When the 1 st second of dynamic activity is detected, the algorithm counts the number of peak chest angles. If the number of peaks is less than or equal to 3 and the maximum angle difference from the mean angle of last second of static activity is greater than $20^{\circ}$, a transition is detected. If the number of peaks is greater than 3 , the algorithm estimates the power spectrum density (PSD) of the original input chest angles to identify the step frequency. If the step frequency falls in the range obtained from the training data [15], locomotion is detected; otherwise that second of time will be labeled as an unidentified dynamic. The algorithm processes the activity identification sequence using a window of 1 second and provides a summary of total durations of each activity for the quantification of activity level.

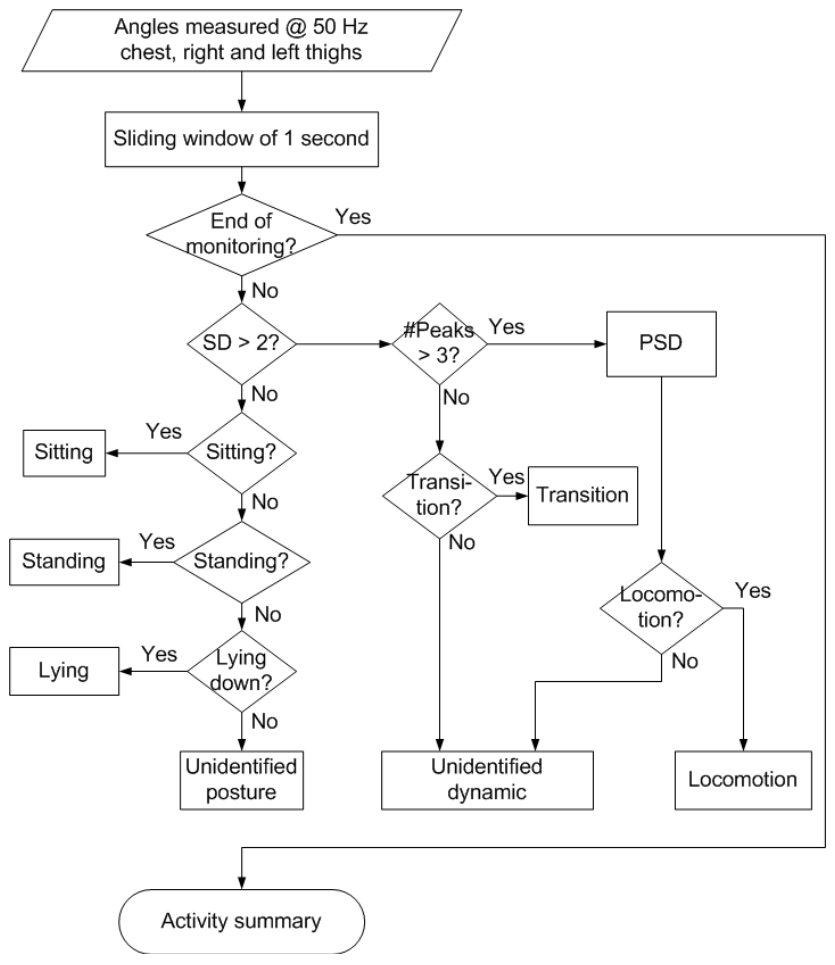

Figure 3. Activity recognition algorithm flowchart 


\section{RESULTS}

1) First study results: The measurements of static angle are shown and compared in Table I. The difference in mean scores per position between the FAM system and the Optotrak system ranges from minimally $0.26^{\circ}$ to maximally $1.18^{\circ}$ with a mean difference of $0.57^{\circ}$ and a SD of $0.92^{\circ}$ across all positions.

2) Second study results: Descriptive results of measuring oscillation amplitudes are compared in Fig. 4, and the error bars indicate the $95 \%$ confidence interval. The difference between the FAM system and the Optotrak system ranges from minimally $0.06^{\circ}$ to maximally $2.3^{\circ}$ with an average of $0.601^{\circ}$ and a SD of $0.188^{\circ}$ across all oscillations.

3) Third study results: The agreement of activity recognition between the FAM system and the video recordings is $98.96 \%$ in average for all static postures, transitions and locomotion. The Sensitivity (probability of detection) of each activity is shown in Table II with a maximum of $100 \%$ and a minimum of $96.2 \%$. The mean difference in the durations of the activities between the FAM system and the video recordings is 0.148 second and the $\mathrm{SD}$ is 0.235 second.

\section{TABLE I. FIST STUDY COMPARISON}

\begin{tabular}{|l|r|r|r|r|r|}
\hline Protractor & 0 & 30 & 60 & 90 & $120(-60)$ \\
\hline FAM & 0.801 & 32.503 & 64.671 & 91.108 & -59.837 \\
\hline Optotrak & 1.985 & 33.65 & 64.409 & 90.188 & -58.654 \\
\hline
\end{tabular}

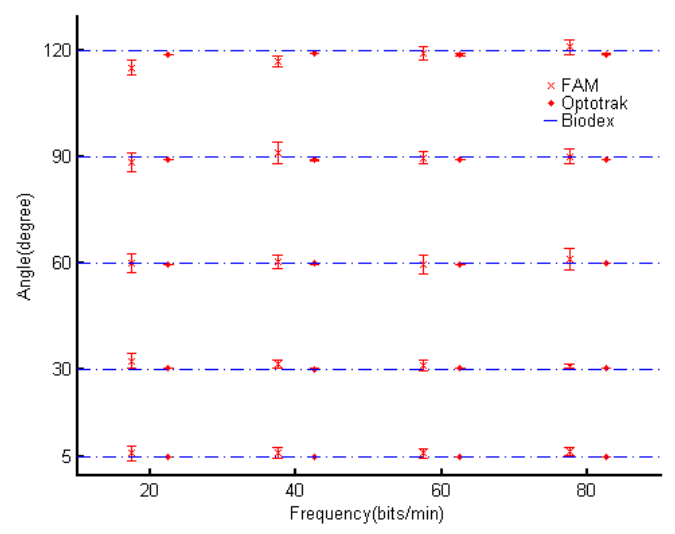

Figure 4. Activity recognition algorithm flowchart

TABLE II. SENSITIVY OF ACTIVITY RECOGNITION

\begin{tabular}{|l|c|c|c|c|c|}
\hline \multirow{2}{*}{ activity } & \multicolumn{3}{|c|}{ postures } & \multirow{2}{*}{ locomotion } & transitions \\
\cline { 2 - 4 } & sitting & standing & lying & & \\
\hline sensitivity & $98.60 \%$ & $96.2 \%$ & $100 \%$ & $100 \%$ & $100 \%$ \\
\hline
\end{tabular}

\section{DISCUSSION}

In this paper, we have presented computationally efficient algorithms for measuring angles and monitoring functional activities using kinematic data from three wearable wireless miniaturized FAM sensors that can be conveniently worn by elderly people and patients with chronic pains, Parkinson's disease, and risk of stroke, and a smartphone that will be widely used in the whole society. The signal processing algorithm proposed in the FAM system is shown to have high accuracy on a data set for measuring both static and dynamic angles. The activity recognition algorithm is shown to have consistently high sensitivity for general functional activities such as postures, locomotion and transitions in all trials. In our on-going research, we are implementing these algorithms on the smartphone for real-time online monitoring and testing the FAM system on subjects for longer time, such as a day or a week, in the home and community settings. In future research, we will assess the performance of the other two axes, work on a hierarchical configuration of the FAM system and develop a stochastic decision algorithm for free-style monitoring. We also will extend to develop smartspaces for pervasive health.

\section{REFERENCES}

[1] B. C. Spillamn and J. Lubitz, "The effect of longetivity on spending for acute and leong-term care," N. Eng. J. Med., vol. 342, pp. 1409-1415, 2000 .

[2] U.S. Bureau of the Census, Population Distrubution in 2005.

[3] K. Aminian, A. Paraschiv-lonescu, E. E. Buchser, R. Rutschmann, M. Depairon, and P. Robert, "Ambulatory system for gait and posture analysis: validation in chronic pain patients treated by spinal cord stimulation," International Soc. for Postural and Gait Research Symposium (ISPGR), Sydney, Australia, March 2003, pp. 90.

[4] F. M. Gloth IIIrd, J. Walston, J. Meyer, and J. Pearson, "Reliability and validity of the Frail elderly functional assessment questionnaire," Am J Phys Med Rehabil, vol. 74, pp. 45-53, 1995.

[5] B. Kopp, A. Kunkel, H. Flor, T. Platz, U. Rose, K. H. Mauritz, K. Gresser, K. L. McCulloch, and E. Taub, "The arm motor ability test: reliability, validity, and sensitivity to change of an instrument for assessing disbilities in activities of daily living," Arch. Phys. Med. Rehabil, vol. 78, pp. 615-620, 1997.

[6] M. N. Nyan, F. E. H. Tay, and M. Z. E. Mah, "Application of motion analysis system in pre-impact fall detection," J Biomechanics, vol. 41, pp. 2297-2304, 2008.

[7] M. C. Boonstra, R. M. Van Der Slikke, N. L. Keijsers, R. C. Van Lummel, M. C. De Waal Malefijt, and N. Verdonschot, "The accuracy of measuring the kinematics of rising from a chair with accelerometers and gyroscopes," J Biomechanics, vol. 39 (2), pp. 354-358, 2006.

[8] H. J. Luinge, P. H. Veltink, and C. T. Baten, "Estimating orientation with gyroscopes and accelerometers," Technology and Healthcare, pp. 455-459, 1999

[9] Q. Liu, and J. Teng, "Reseach of gyro signal de-noise with stationary wavelets transform," CCECE, 2003, pp 1989-1992.

[10] X. Chen, "Modeling random gyro drift by time series neural networks and by traditional method," Proc. Of IEEE Int Conf Neural Networks and signal processing, Nanjing, China, pp. 810-813, Dec. 2003.

[11] R. A. States, and E. Pappas, "Precision and repeatability of the Optotrak 3020 motion measurement system," J. Med. Eng. Tech, pp. 11-16, 2006.

[12] J. M. Druin, T. C. Valovich-mcLeod, S. J. Shultz, B. M. Gansneder, and D. H. Perrin, "Reliability and validity of the Biodex system 3 pro isokinetic dynamometer velocity, torque and position measurements," Eur J Appl Physiol, pp. 22-29, 2003.

[13] M. Lyons, K. M. Culhane, D. Hilton, P. A. Grace PA, and D. Lyons, "A description of an accelerometer-based mobility monitoring technique," Med Eng Phys, vol. 27(6), pp. 497-504, 2005.

[14] X. Ji, S. Wang, Y. Xu, Q. Shi, and D. Xia, "Application of the digital signal processing in the MEMS gyroscope de-drift," proc of 1st IEEE international conf on Nano/Micro Engineered and Molecular Systems, Zhuhai, China, Jan 2006.

[15] R. C. Wagenaar, and R. E. A van Emmerik, "Resonant frequencies of arms and legs identify different walking patterns", J of Biomechanics, vol. 33 , pp. $853-861,2000$. 